\title{
MAGNETIC RESONANCE IMAGING IN ACUTE HEPATIC ENCEPHALOPATHY
}

\author{
Mathew C. Koshy'1, Naufal Perumpalath², Rajendran V. R3 , Jineesh $T^{4}$, Saanida M. P5, Juvaina P6, Gomathy Subramanyam ${ }^{7}$, Rajan P8
}

1 Junior Resident, Department of Radiology, Government Medical College, Calicut.

${ }^{2}$ Associate Professor, Department of Radiology, Government Medical College, Calicut.

3Professor, Department of Radiology, Government Medical College, Calicut.

${ }^{4}$ Assistant Professor, Department of Radiology, Government Medical College, Calicut.

${ }_{5}^{5}$ Assistant Professor, Department of Radiology, Government Medical College, Calicut.

${ }^{6}$ Assistant Professor, Department of Radiology, Government Medical College, Calicut.

${ }^{7}$ Additional Professor, Department of Radiology, Government Medical College, Calicut.

${ }^{8}$ Professor, Department of Radiology, Government Medical College, Calicut.

\section{ABSTRACT}

\section{AIMS AND OBJECTIVES}

To study the pattern of brain involvement in acute hepatic encephalopathy in patients with chronic liver disease and correlate the extent of involvement on magnetic resonance imaging with serum ammonia and clinical severity.

\section{MATERIALS AND METHODS}

Thirty patients with clinical or radiological evidence of chronic liver disease who presented with acute onset neuropsychiatric symptoms with a clinical diagnosis of acute hepatic encephalopathy. A detailed history was taken. Status of chronic liver disease confirmed, serum ammonia values estimated and magnetic resonance imaging done within 7 days of admission. The anatomic pattern of involvement in FLAIR and DWI (comparison with ADC maps) noted. The initial clinical severity (assessed by West Haven grade) and serum ammonia values were recorded and correlated with extent of involvement in magnetic resonance imaging. The patients were followed up for 21 days from the day of MRI to get an idea about inpatient or outpatient status; however, no clinical or radiological end points were evaluated.

\section{OBSERVATION AND RESULTS}

On FLAIR and DWI, there were abnormalities in PLIC (73.3\%, 60\%), thalamus (63.3\%, 53.3\%), PVWM $(26.7 \%, 16.7 \%)$ and IC $(36.7 \%, 23.3 \%)$. There was significant correlation $(\mathrm{P}<0.05)$ for $\mathrm{PVWM}(\mathrm{P}=0.001,0.002)$ and $\mathrm{IC}(\mathrm{P}=0.005,0.009)$ involvement with clinical severity on FLAIR and DWI. There was no significant correlation for PLIC $(\mathrm{P}=0.371,0.162)$ and thalamic $(\mathrm{P}=0.252,0.92)$ involvement. All the patients showed elevated serum ammonia values and a period of inpatient stay $>21$ days.

\section{CONCLUSION}

The constellation of anatomic involvement in the appropriate clinical setting is suggestive of acute hepatic encephalopathy. Involvement of PVWM and IC indicated a higher grade of initial clinical severity.

\section{KEYWORDS}

Hepatic encephalopathy, FLAIR, Diffusion Restriction, West Haven Grade.

HOW TO CITE THIS ARTICLE: Koshy MC, Perumpalath N, Rajendran VR, et al. Magnetic resonance imaging in acute hepatic encephalopathy. J. Evolution Med. Dent. Sci. 2016;5(77):5741-5745, DOI: 10.14260/jemds/2016/1294

\section{INTRODUCTION}

Acute hepatic encephalopathy is a reversible neuropsychiatric condition of major clinical significance that complicates endstage liver disease. Because of the compromised liver function, the brain is exposed to a number of potential neurotoxins like ammonium ion, glutamine, mercaptan, Octopamine, phenolic compounds, gamma-aminobutyric acid.(1) It affects the cerebral metabolism, blood brain barrier integrity and sodium-potassium adenosine triphosphatase function.(2) Subtle signs of hepatic encephalopathy are observed in $70 \%$ of the patients with cirrhosis.(3),(4)

Financial or Other, Competing Interest: None.

Submission 15-07-2016, Peer Review 06-08-2016,

Acceptance 13-08-2016, Published 26-09-2016.

Corresponding Author:

Dr. Mathew C. Koshy,

Puthenveetil Medayil

Thevalakkara P. $O$.

Kollam-690524.

E-mail: drmathewckoshy@gmail.com

DOI: $10.14260 /$ jemds/2016/1294
The symptoms may be debilitating in a significant number of patients. Overt hepatic encephalopathy occurs in $30-45 \%$ of patients with cirrhosis. (5)

It is also observed in $24-53 \%$ of patients who undergo portosystemic shunt surgery. The development of hepatic encephalopathy negatively impacts patient survival.(6) The development of hepatic encephalopathy severe enough to lead to hospitalisation is associated with a survival probability of $42 \%$ at 1 year and $23 \%$ at 3 years. Approximately, $30 \%$ of the patients dying of end-stage liver disease experience significant encephalopathy approaching coma.

The estimated prevalence of chronic liver disease and cirrhosis in the United States is approximately 5.5 million cases. In 2003, there were over 40000 patients hospitalised in United States for a primary diagnosis of hepatic encephalopathy, resulting in total charges of approximately $\$ 932$ million. Furthermore, trends over the past 10 years suggest that the burden of hepatic encephalopathy is increasing, as indicated by increases in hospital admissions and higher charges per stay.(7) 
The diagnosis of hepatic encephalopathy is basically clinical and the role of imaging equivocal. Computed tomography evaluation of brain has been non-specific with most of the studies being normal or showing age related changes in brain parenchyma.

All magnetic resonance imaging studies have been directed at chronic hepatic encephalopathy which has revealed hyperintensity on T1 weighted images in the basal ganglia. There are very few studies which were done for the evaluation of symptoms of hepatic encephalopathy in an acute setting. There has been almost no studies undertaken in Asia or India for the same. However, recent studies have indicated that a diagnosis of acute hepatic encephalopathy can be made on magnetic resonance imaging. Therefore, with this background this study is aimed at assessing whether the described findings are reproducible. An attempt is being made to categorise and correlate, the extent of involvement on magnetic resonance imaging and clinical severity based on West-Haven criteria. The sequences being used are FLAIR (Fluid Attenuated Inversion Recovery) and DWI (Diffusion Weighted Imaging).

\section{AIMS AND OBJECTIVES}

1. To study the pattern of brain involvement in acute hepatic encephalopathy in patients with chronic liver disease.

2. To correlate the extent of involvement on magnetic resonance imaging with serum ammonia and clinical severity.

\section{MATERIALS AND METHODS}

- Study Design: Descriptive study.

- Study Setting: Government Medical College, Kozhikode.

- Study Period: 12 months from March 2014 to June 2015.

- Sample Size: All patients referred to the Department of Radiodiagnosis, Kozhikode will be studied, subject to patient consent for being included in the same.

- Research Subject: Patients with chronic liver disease who present with acute onset neuropsychiatric symptoms with a clinical diagnosis of acute hepatic encephalopathy.

\section{Inclusion Criteria}

- Clinically or radiologically proven cases of chronic liver disease with neuropsychiatric symptoms undergoing MR imaging in the Department of Radiodiagnosis, Medical College, Calicut.

- Only patients between 20-80 years of age will be included in the study.

\section{Exclusion Criteria}

- Patients with associated electrolyte disturbances.

- Patients not consenting to be part of the study were excluded from the study.

\section{METHODOLOGY}

\section{Diagnostic Workup}

Patients were selected based on inclusion and exclusion criteria. A detailed history was taken. Status of chronic liver disease confirmed and serum ammonia values and magnetic resonance imaging estimated within 7 days of admission.

\section{Technique}

Magnetic resonance imaging done with a GE Signa 1.5T HDxt, 16-channel scanner using a standard brain protocol-T1, T2, FLAIR (fluid attenuated inversion recovery), GRE (gradient echo) and DWI (diffusion weighted imaging). FLAIR and DWI will be evaluated for the study. All patients were studied in the first week. FLAIR is an inversion recovery sequence to suppress CSF, which is highly sensitive for detecting periventricular lesions as well as oedema. Diffusion is an imaging sequence which utilises the reduction in random diffusion of water molecules as a result of cytotoxic oedema. The presence or absence of diffusion restriction would be evaluated by comparing with ADC (Apparent diffusion coefficient) maps. The presence of hypointensity on ADC maps corresponding to diffusion hyperintensity will be taken as showing diffusion restriction. The presence of hyperintensity on ADC maps will be considered as T2 shine through. A gradient strength of $b=1000 \mathrm{~s} / \mathrm{mm}^{2}$ was used for DWI. The reason for selecting FLAIR and DWI sequences for the study was by keeping under consideration the pathophysiology of cerebral changes in acute hepatic encephalopathy, i.e. cerebral oedema.

The section thickness was $10 \mathrm{~mm}$ for each sequence. Then, patients were followed up for a period of 3 weeks, from the date of magnetic resonance imaging which corresponded to hospital stay for all patients.

- Data Collection Tools: Structured data collection proforma.

- Data Analysis: All the raw data was consolidated into a master chart based on the anatomical area of involvement in FLAIR and DWI. The data was analysed as to evolve a grading based pattern in comparison to clinical severity. Data analysis was done with the help of Microsoft Excel 2010 Worksheet and SPSS 13 statistical software.

\section{OBSERVATION AND RESULT}

\begin{tabular}{|c|c|c|}
\hline & & Acute Hepatic Encephalopathy \\
\hline \multirow{4}{*}{ Age } & $41-50$ & 8 \\
\cline { 2 - 3 } & $51-60$ & 12 \\
\cline { 2 - 3 } & $61-70$ & 10 \\
\cline { 2 - 3 } & Total & $\mathbf{3 0}$ \\
\hline \multicolumn{2}{|c|}{ Table 1: Age Distribution of Patients } \\
\hline
\end{tabular}

Mean was 55.9 years with a standard deviation of 7.177 .

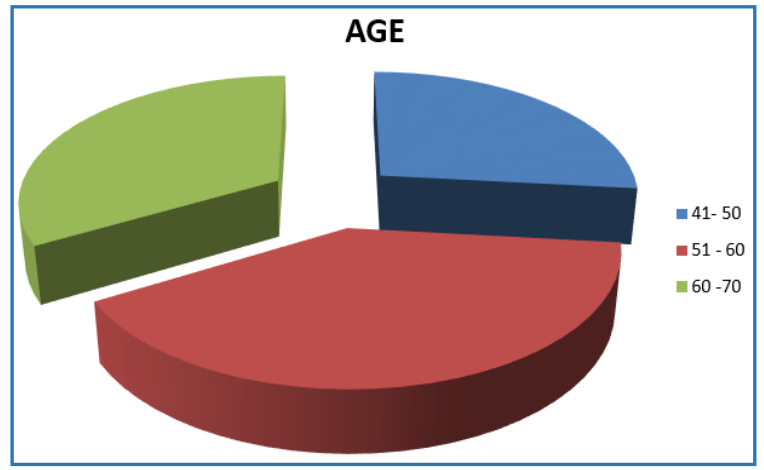

Chart 1: Age Distribution of Patients 


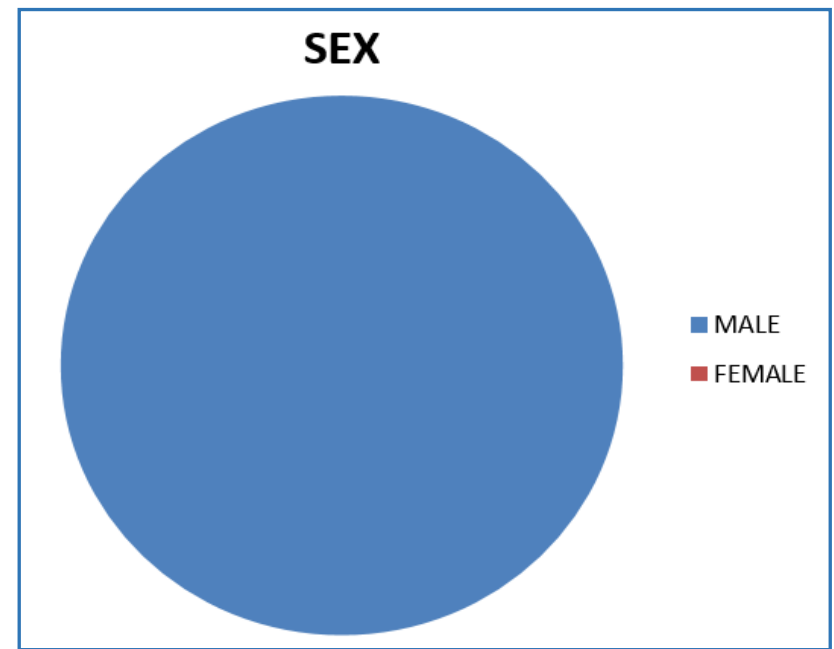

Chart 2: Sex Distribution of Patients

All the patients studied were males.

\begin{tabular}{|c|c|c|}
\hline West Haven Grade & Frequency & Percentage \\
\hline 1 & 11 & 36.7 \\
\hline 2 & 15 & 50.0 \\
\hline 3 & 4 & 13.3 \\
\hline 4 & 0 & 0 \\
\hline Total & $\mathbf{3 0}$ & $\mathbf{1 0 0}$ \\
\hline \multicolumn{3}{|c|}{$\begin{array}{c}\text { Table 2: Distribution of Patients Based } \\
\text { on West Haven Grade }\end{array}$} \\
\hline
\end{tabular}

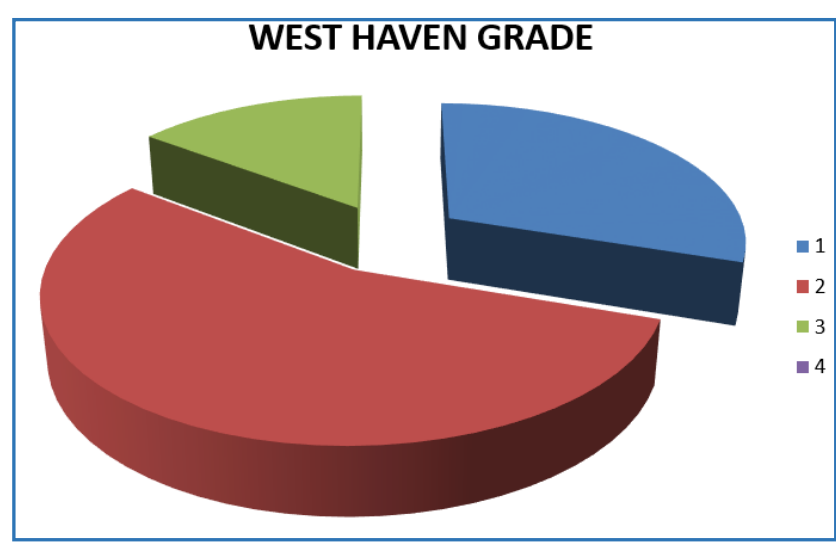

Chart 3: West Haven Grade

\begin{tabular}{|c|c|c|c|c|c|}
\hline & \multicolumn{5}{|c|}{ FLAIR Hyperintensity } \\
\hline & PLIC & THALAMUS & PVWM & IC & DCI \\
\hline Yes & 22 & 19 & 8 & 11 & 0 \\
\hline No & 8 & 11 & 22 & 19 & 30 \\
\hline \multicolumn{5}{|c|}{ Table 3: Distribution of FLAIR Hyperintensity } \\
\hline
\end{tabular}

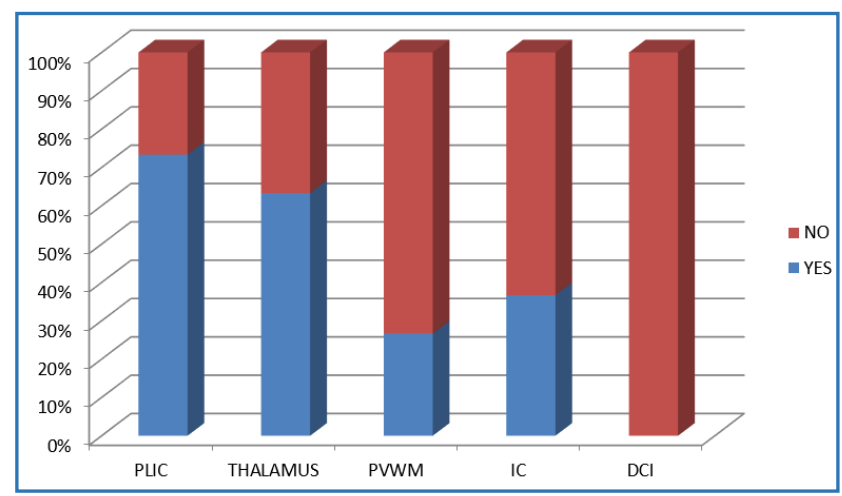

Chart 4: Distribution of FLAIR Hyperintensity

\begin{tabular}{|c|c|c|c|c|c|}
\hline & \multicolumn{5}{|c|}{ Diffusion Restriction } \\
\hline & PLIC & Thalamus & PVWM & IC & DCI \\
\hline Yes & 18 & 16 & 5 & 7 & 0 \\
\hline No & 12 & 14 & 25 & 23 & 30 \\
\hline \multicolumn{5}{|c|}{ Table 4: Distribution of Diffusion Restriction } \\
\hline
\end{tabular}

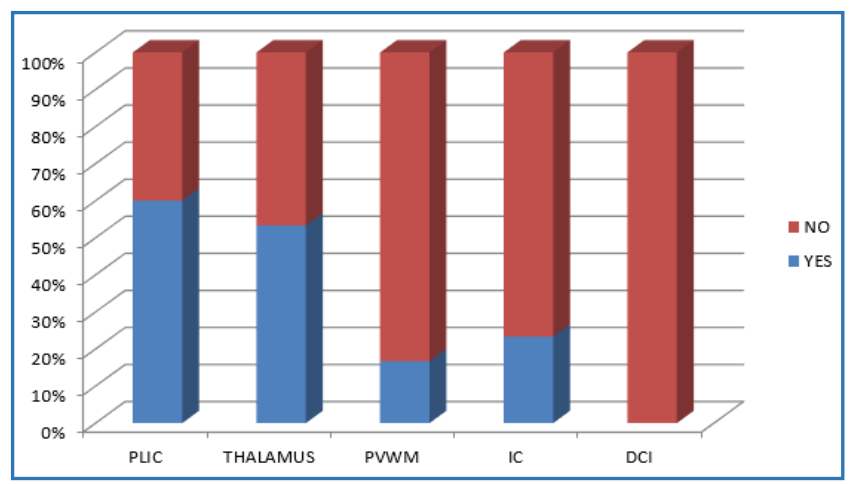

Chart 5: Distribution of Diffusion Restriction

\begin{tabular}{|c|c|c|}
\hline & Frequency & Percent \\
\hline Valid & 30 & 100.0 \\
\hline \multicolumn{2}{|c|}{ Table 5: Frequency of Elevated Ammonia Levels } \\
\hline
\end{tabular}

\begin{tabular}{|c|c|c|}
\hline & Frequency & Percent \\
\hline Valid & 30 & 100.0 \\
\hline \multicolumn{2}{|c|}{ Table 6: Frequency of Patients with $>3$} \\
Weeks Hospital Stay
\end{tabular}

\begin{tabular}{|c|c|c|c|c|}
\hline \multirow{2}{*}{} & \multicolumn{3}{|c|}{ West Haven Grade } & \multirow{2}{*}{ Total } \\
\cline { 2 - 4 } & $\mathbf{1}$ & $\mathbf{2}$ & $\mathbf{3}$ & \\
\hline FLAIR & 7 & 11 & 4 & 22 \\
Hyperintensity in & $31.8 \%$ & $50.0 \%$ & $18.2 \%$ & $100 \%$ \\
\cline { 2 - 5 } PLIC & 4 & 4 & 0 & 8 \\
-Yes & $50.0 \%$ & $50.0 \%$ & 0 & $100 \%$ \\
-No & 11 & 15 & 4 & 30 \\
& $36.7 \%$ & $50.0 \%$ & $13.3 \%$ & $100 \%$ \\
\hline
\end{tabular}

Table 7: Distribution of FLAIR Hyperintensity in Posterior Limb of Internal Capsule in Comparison to West Haven Criteria

Chi-Square test was applied. P value obtained was 0.371 , was found to be not significant.

\begin{tabular}{|c|c|c|c|c|}
\hline \multirow{2}{*}{} & \multicolumn{3}{|c|}{ West Haven Grade } & \multirow{2}{*}{ Total } \\
\cline { 2 - 5 } & $\mathbf{1}$ & $\mathbf{2}$ & $\mathbf{3}$ & 19 \\
FLAIR & 6 & 9 & 4 & 19 \\
Hyperintensity in & $31.6 \%$ & $47.4 \%$ & $21.1 \%$ & $100 \%$ \\
\cline { 2 - 5 } Thalamus - Yes & 5 & 6 & 0 & 11 \\
-No & $45.5 \%$ & $54.5 \%$ & 0 & $100 \%$ \\
\hline & 11 & 15 & 4 & 30 \\
& $36.7 \%$ & $50.0 \%$ & $13.3 \%$ & $100 \%$ \\
\hline Table 8: Distribution of Thalamic Hyperintensity in \\
FLAIR in Comparison to West Haven Grade \\
\hline
\end{tabular}

Chi-Square test was applied and the P value obtained was 0.252 . Thus not significant. 


\begin{tabular}{|c|c|c|c|c|}
\hline & \multicolumn{3}{|c|}{ West Haven Grade } & \multirow[b]{2}{*}{ Total } \\
\hline & 1 & 2 & 3 & \\
\hline \multirow{3}{*}{$\begin{array}{l}\text { FLAIR } \\
\text { Hyperintensity in } \\
\text { PVWM - Yes }\end{array}$} & 0 & 4 & 4 & 8 \\
\hline & $0 \%$ & $50.0 \%$ & $50.0 \%$ & $100 \%$ \\
\hline & 11 & 11 & 0 & 22 \\
\hline \multirow[t]{2}{*}{-No } & $50.0 \%$ & $50.0 \%$ & 0 & $100 \%$ \\
\hline & $\begin{array}{c}11 \\
36.7 \%\end{array}$ & $\begin{array}{c}15 \\
50.0 \%\end{array}$ & $\begin{array}{c}4 \\
13.3 \%\end{array}$ & $\begin{array}{c}30 \\
100 \%\end{array}$ \\
\hline \multicolumn{5}{|c|}{$\begin{array}{c}\text { Table 9: Distribution of FLAIR Hyperintensity in } \\
\text { Periventricular White Matter in Comparison } \\
\text { to West Haven Grade }\end{array}$} \\
\hline
\end{tabular}

Chi-Square test was applied, $\mathrm{P}$ value calculated was 0.001 which was significant.

\begin{tabular}{|c|c|c|c|c|}
\hline \multirow{2}{*}{} & \multicolumn{3}{|c|}{ West Haven Grade } & \multirow{2}{*}{ Total } \\
\cline { 2 - 4 } & $\mathbf{1}$ & $\mathbf{2}$ & $\mathbf{3}$ & \\
\hline FLAIR & 0 & 8 & 3 & 11 \\
Hyperintensity in & $0 \%$ & $72.7 \%$ & $27.3 \%$ & $100 \%$ \\
\cline { 2 - 5 } $\begin{array}{c}\text { Insular Cortex - } \\
\text { Yes }\end{array}$ & 11 & 7 & 1 & 19 \\
& $57.9 \%$ & $36.8 \%$ & $5.3 \%$ & $100 \%$ \\
-No & & & & \\
\hline Total & $\mathbf{1 1}$ & $\mathbf{1 5}$ & $\mathbf{4}$ & $\mathbf{3 0}$ \\
& $\mathbf{3 6 . 7 \%}$ & $\mathbf{5 0 . 0} \%$ & $\mathbf{1 3 . 3 \%}$ & $\mathbf{1 0 0} \%$ \\
\hline
\end{tabular}

Table 10: Distribution of FLAIR Hyperintensity in Insular Cortex in Comparison to West Haven Grade

Chi-Square was applied, $\mathrm{P}$ value was calculated as 0.005 which was significant.

\begin{tabular}{|c|c|c|c|c|}
\hline \multirow{2}{*}{} & \multicolumn{3}{|c|}{ West Haven Grade } & \multirow{2}{*}{ Total } \\
\cline { 2 - 5 } $\begin{array}{c}\text { Restriction in PLIC } \\
\text {-Yes }\end{array}$ & 5 & $\mathbf{2}$ & $\mathbf{3}$ & 18 \\
& $27.8 \%$ & $50.0 \%$ & $22.2 \%$ & $100 \%$ \\
\cline { 2 - 5 } -No & 6 & 6 & 0 & 12 \\
Total & 11 & 15 & 4 & 30 \\
& $36.7 \%$ & $50.0 \%$ & $13.3 \%$ & $100 \%$ \\
\hline Table 11: Distribution of Diffusion Restriction in \\
Posterior Limb of Internal Capsule in \\
Comparison to West Haven Grade \\
\hline
\end{tabular}

Chi-Square test applied, and $\mathrm{P}$ value calculated as 0.162 , which was not significant.

\begin{tabular}{|c|c|c|c|c|}
\hline \multirow{2}{*}{} & \multicolumn{3}{|c|}{ West Haven Grade } & \multirow{2}{*}{ Total } \\
\cline { 2 - 5 } & $\mathbf{1}$ & $\mathbf{2}$ & $\mathbf{3}$ & 16 \\
Diffusion & 4 & 8 & 4 & $16 \%$ \\
Restriction in & $25.0 \%$ & $50.0 \%$ & $25.0 \%$ & $100 \%$ \\
\cline { 2 - 5 } Thalamus - Yes & 7 & 7 & 0 & 14 \\
-No & $50.0 \%$ & $50.0 \%$ & $0 \%$ & $100 \%$ \\
\hline Total & $\mathbf{1 1}$ & $\mathbf{1 5}$ & $\mathbf{4}$ & $\mathbf{3 0}$ \\
& $\mathbf{3 6 . 7} \%$ & $\mathbf{5 0 . 0} \%$ & $\mathbf{1 3 . 3 \%}$ & $\mathbf{1 0 0 \%}$ \\
\hline Table 12: Distribution of Diffusion Restriction in \\
Thalamus in Comparison to West Haven Grade \\
\hline
\end{tabular}

Chi-Square test applied, P value was calculated as 0.092, which was not significant.

\begin{tabular}{|c|c|c|c|c|}
\hline & \multicolumn{3}{|c|}{ West Haven Grade } & \multirow{2}{*}{ Total } \\
\cline { 2 - 5 } & $\mathbf{1}$ & $\mathbf{2}$ & $\mathbf{3}$ & 5 \\
Destriction in & 0 & 2 & 3 & 5 \\
PVWM - Yes & $0 \%$ & $40.0 \%$ & $60.0 \%$ & $100 \%$ \\
\cline { 2 - 5 } & 11 & 13 & 1 & 25 \\
-No & $44.0 \%$ & $52.0 \%$ & $4 \%$ & $100 \%$ \\
\hline Total & 11 & 15 & 4 & 30 \\
& $36.7 \%$ & $50.0 \%$ & $13.3 \%$ & $100 \%$ \\
\hline Table 13: Distribution of Diffusion Restriction in \\
Periventricular White Matter in Comparison \\
to West Haven Grade \\
\hline
\end{tabular}

Chi-Square test applied, $\mathrm{P}$ value was calculated as 0.002 , which was significant.

\begin{tabular}{|c|c|c|c|c|}
\hline \multirow{2}{*}{} & \multicolumn{3}{|c|}{ West Haven Grade } & \multirow{2}{*}{ Total } \\
\cline { 2 - 5 } & $\mathbf{1}$ & $\mathbf{2}$ & $\mathbf{3}$ & 7 \\
Diffusion & 0 & 4 & 3 & 7 \\
Restriction in \\
Insular Cortex - Yes & $0 \%$ & $57.1 \%$ & $42.9 \%$ & $100 \%$ \\
\cline { 2 - 5 } & 11 & 11 & 1 & 23 \\
-No & $47.8 \%$ & $47.8 \%$ & $4.3 \%$ & $100 \%$ \\
\hline Total & 11 & 15 & 4 & 30 \\
& $36.7 \%$ & $50.0 \%$ & $13.3 \%$ & $100 \%$ \\
\hline Table 14: Distribution of Diffusion Restriction in Insular \\
Cortex in Comparison to West Haven Grade \\
\hline
\end{tabular}

Chi-square test applied, P value was calculated as 0.009 , which was significant. Cross table evaluation of diffuse cortical involvement (DCI) was not done as none of the patients showed this particular pattern.

\section{DISCUSSION}

Acute hepatic encephalopathy is essentially a clinical diagnosis. Evaluation of serum ammonia levels and clinical grading is part of routine workup. However, neither of them have shown significant correlation. The purpose of radiological evaluation has so far been limited to CT evaluation of head to rule secondary causes.

In this study, an attempt has been made to identify anatomical patterns of involvement of brain using FLAIR and DWI. Based on a study by McKinney et al(8), five anatomical areas were identified-posterior limb of internal capsule, thalamus, insular cortex, periventricular white matter and diffuse cortical involvement. Each of these sites were evaluated for all patients and the extent of involvement on magnetic resonance imaging and correlated with initial clinical severity. All the patients showed varying involvement of the above said anatomical regions and no other new area of involvement was noted in the present study. The areas of involvement corresponded with the study conducted by McKinney et al. However, diffuse cortical involvement noted in 6 out of the 20 patients studied by McKinney et al was not seen in the present study. Therefore, it may be inferred that the constellation of these findings is suggestive of acute hepatic encephalopathy.

On applying statistical tests to establish the significance of the obtained results, it was found that FLAIR hyperintensity and diffusion restriction in posterior limb of internal capsule and thalamus did not show any significant association, which is comparable with the study by McKinney et al. The frequency of distribution of FLAIR hyperintensity and diffusion restriction in posterior limb of internal capsule and thalamus 
were noted across all clinical grades with more percentage of patients in latter grades showing the same.

However, the FLAIR hyperintensity and diffusion restriction in periventricular white matter and insular cortex showed significant $P$ values on analysis with West Haven grade. The frequency of distribution of FLAIR hyperintensities and diffusion restriction in periventricular white matter and insular cortex were noted to more often indicate a clinical grade of 2 or 3 , with almost all patients with clinical grade 3 showing the above pattern. None of the patients with clinical grade 1 showed FLAIR hyperintensity in periventricular white matter and insular cortex. This was in contrast with the study by McKinney et al for whom there were no significant associations.

All the patients included in the study showed elevated serum ammonia levels and the absolute values showed no correlation with the clinical grades apart from being abnormal.

All patients were followed up for a period of three weeks from the day of magnetic resonance imaging. All patients were still under admission and inpatient care and none of the patients irrespective of the clinical severity were discharged for outpatient care.

In Light of the above findings, an Arbitrary Magnetic Resonance Imaging based Classification may be Inferred

Grade 1: Bilateral posterior limb of internal capsule or thalamic involvement.

Grade 2: Bilateral posterior limb of internal capsule with thalamus involvement.

Grade 3: Bilateral posterior limb of internal capsule with thalamus periventricular white matter and insular cortex involvement.

Grade 4: Grade 3 with diffuse cortical involvement/latter alone.

Grade 1 was assigned as thalamus or posterior limb of internal capsule involvement because most of the West Haven grade 1 patients had either of the two with no specific predilection for either. The confidence in assigning grade 4 is less than that with other grades, as no patients with clinical grade 4 was imaged and diffuse cortical involvement was not seen in any of the patients in the present study.

\section{Limitations of the Study}

1. Sample size is small.

2. The presence of diffusion restriction was evaluated by visual impression with ADC maps and absolute ADC values were not calculated.

3. Although the patients were followed up, only inpatient/outpatient status was considered, no clinical or radiological status was evaluated.

\section{CONCLUSION}

- Magnetic resonance imaging is the only imaging tool that can be used to demonstrate the spectrum of brain involvement.
- The constellation of anatomical involvement including posterior limb of internal capsule, thalamus, periventricular white matter and insular cortex in the appropriate clinical setting is suggestive of acute hepatic encephalopathy.

- Progressive involvement periventricular white matter and insular cortex tends to indicate a higher clinical grade at evaluation.

- However, the proposed magnetic resonance imaging grading is arbitrary and more definitive evidence has to be accumulated with a larger sample and radiological followup.

\section{ABBREVIATIONS}

FLAIR: Fluid attenuated inversion recovery.

DWI: Diffusion weighted imaging.

ADC: Apparent diffusion co-efficient.

PLIC: Posterior limb of internal capsule.

PVWM: Periventricular white matter.

IC: Insular cortex.

MR: Magnetic resonance imaging.

\section{REFERENCES}

1. Al Mardini H, Bartlett $\mathrm{K}$, Record CO. Blood and brain concentrations of mercaptans in hepatic and methanethiol induced coma. Gut 1984;25(3):284-90.

2. Butterworth RF. Pathogenesis of hepatic encephalopathy: update on molecular mechanisms. Indian J Gastroenterol 2003;22(Suppl 2):S11-6.

3. Amodio P, Montagnese S, Gatta A, et al. Characteristics of minimal hepatic encephalopathy. Metab Brain Dis 2004;19(3-4):253-67.

4. Hartmann IJ, Groeneweg M, Quero JC, et al. The prognostic significance of subclinical hepatic encephalopathy. Am J Gastroenterol 2000;95(8):2029-34.

5. Hass HG, Hösel F, Mader I, et al. Subclinical and overt hepatic encephalopathy-detection and treatment control by proton magnetic resonance spectroscopy (1H-MRS). In: Jones EA, Meijer A, Chamuleau RFM, eds. Encephalopathy and nitrogen metabolism in liver failure se-13. Netherlands: Springer 2003:125-33.

6. Gerber T, Schomerus H. Hepatic encephalopathy in liver cirrhosis: pathogenesis, diagnosis and management. Drugs 2000;60(6):1353-70.

7. Poordad FF. Review article: the burden of hepatic encephalopathy. Aliment Pharmacol Ther 2007;25(Suppl 1):3-9.

8. McKinney AM, Lohman BD, Sarikaya B, et al. Acute hepatic encephalopathy: diffusion-weighted and fluid-attenuated inversion recovery findings and correlation with plasma ammonia level and clinical outcome. AJNR Am J Neuroradiol 2010;31(8):1471-9. 